\title{
Original
}

\section{Lifestyle and osteoporosis in middle-aged and elderly women: Chiba bone survey}

\author{
Ichiro Tatsuno ${ }^{1)}$, Takashi Terano ${ }^{2)}$, Mitsugu Nakamura ${ }^{3)}$, Kiminori Suzuki ${ }^{4)}$, Kazuko Kubota ${ }^{5)}$, \\ Jyunichi Yamaguchi ${ }^{5)}$, Tomohiko Yoshida ${ }^{6)}$, Sawako Suzuki ${ }^{6}$, Tomoaki Tanaka $^{6)}$ and Makio Shozu ${ }^{7)}$ \\ ${ }^{1)}$ Center for Diabetes, Metabolism and Endocrinology, Toho University Sakura Medical Center, Sakura 285-8741, Japan \\ ${ }^{2)}$ Department of Medicine, Chiba Aoba Municipal Hospital, Chiba 260-0852, Japan \\ ${ }^{3)}$ Chiba City Medical Association, Chiba 261-0001, Japan \\ 4) Chiba Foundation for Health Promotion \& Disease Prevention, Chiba 261-0002, Japan \\ ${ }^{5)}$ Chiba City Public Health Office, Chiba 261-0001, Japan \\ ${ }^{6)}$ Department of Clinical Cell Biology, Graduate School of Medicine, Chiba University, Chiba 260-8670, Japan \\ ${ }^{7)}$ Department of Reproductive Medicine, Graduate School of Medicine, Chiba University, Chiba 260-8670, Japan
}

\begin{abstract}
Osteoporosis causes an enormous health and economic impact in Japan. We investigated the relation between lifestyle and bone fracture in middle-aged and elderly women. This was a population-based, multicenter, cross-sectional survey for postmenopausal osteoporosis in Chiba City, Japan (Chiba bone survey). This survey included 64,809 Japanese women aged $>40$ years. All participants underwent anthropometric measurements including bone mineral density (BMD) and completed a structured, nurse-assisted, self-administered questionnaire also including patient lifestyle. Bone fracture during the recent 5 years was observed in $5.3 \%$, and the fracture group had significantly higher age, BMI, and prevalence of delivery, family histories of kyphosis and hip fracture, diabetes mellitus (DM), dyslipidemia, kidney disease, exercise, fall, and osteoporosis, and had significantly lower BMD and proportion of menstruating participants. Logistic regression analysis revealed that bone fracture was closely associated with not only low bone mass but also age, fall, family histories of kyphosis and hip fracture, DM, kidney disease, menopause, and lifestyle factors of dieting, exercise, and alcohol. Women's health care focusing on lifestyle-related fracture risks such as dieting, exercise, and alcohol appears necessary to prevent bone fracture in postmenopausal osteoporosis.
\end{abstract}

Key words: Bone fracture, Osteoporosis, Lifestyle, Dieting behavior, Bone mass

OSTEOPOROSIS is a common disease characterized by a systemic impairment of bone mass, strength, and microarchitecture to increase bone fragility $[1,2]$, and is one of the clinically important pathologic conditions related to long-term estrogen-deficiency in postmenopausal women, especially in the elderly [3, 4]. Osteoporosis causes an enormous health and economic impact on aging populations in the world [5]. Japan has one of the longest life expectancies in the world [6]. The proportion of persons 65 years or older in Japan was $19.5 \%$ in 2005 , and is expected to reach $40.5 \%$ in 2055 [7]. In 2000, the Japanese government enacted a

Submitted Oct. 12, 2012; Accepted Dec. 27, 2012 as EJ12-0368 Released online in J-STAGE as advance publication Jan. 18, 2013

Correspondence to: Ichiro Tatsuno, M.D., Ph.D., Center for Diabetes, Metabolism and Endocrinology, Toho University Sakura Medical Center, 564-1 Shimoshizu, Sakura-City, Chiba 285-8741, Japan. E-mail: ichiro.tatsuno@med.toho-u.ac.jp law to protect women from postmenopausal osteoporosis, in which the medical examination for osteoporosis was recommended to local governments [8].

Obesity is another major concern, in addition to aging, for health care services to face among a number of emerging challenges in the world $[6,9]$, since the increase in life expectancy does not necessarily mean an increase in healthy life, based on the disease burden of increasing chronically ill populations with obesity $[10,11]$. Most diseases increase in elderly people, particularly obesity-related diseases such as type 2 diabetes mellitus (T2DM), coronary heart disease, and mobility problems [10-13]. As a result, there is evidence of social pressure to be thin and, especially for women, of a weight bias [14], and dieting would be a common behavior not only from younger age, but also to middle-aged and elderly populations [12].

Since osteoporotic fragility is known to be related 
to not only low bone mass but also bone quality in relation to women's lifestyle such as alcohol use and T2DM [15], we analyzed women's lifestyle by focusing on bone fracture during the most recent 5 years, and found that bone fracture was closely associated with not only low bone mass but also with a lifestyle comprising dieting behavior, exercise, and alcohol use in addition to T2DM and kidney disease in middle-aged and elderly Japanese women.

\section{Methods}

\section{Study population}

In 2000, the Japanese government revised a law termed "Health and Medical Service Law for the Elderly" [8], in which medical examinations for postmenopausal osteoporosis was recommended to local governments. Based on this law, Chiba City started the Chiba bone survey in cooperation with the Chiba City Medical Association. This was a multicenter, population-based, cross-sectional survey carried out from 2001 at 220 medical clinics in Chiba, Japan. A total of 267,745 women in the age groups of 40 and 50 years between 2001 and 2004, and at 40, 45, 50, 55, 60, 65, and 70 years between 2005 and 2009 (based on resident registrations) received notices of medical examinations for osteoporosis by mail, and 69,837 (26.1\%) of them underwent the examination. In the study, we used only the first-time data of 64,809 women, excluding all data of repeat-examinations. The study was approved by Chiba City Public Health Office, and the Ethics Committee of Sakura Hospital, School of Medicine, Toho University.

\section{Main outcome}

The event of bone fracture during the most recent 5 years was used as criterion, since we studied the relation between recent lifestyle and bone fracture.

\section{Outcome assessment}

All participants underwent anthropometric measurements to calculate body mass index (BMI; weight $(\mathrm{kg}) /$ height $\left.(\mathrm{m})^{2}\right)$, and responded to a structured, nurseassisted, self-administered questionnaire covering domains that included patient characteristics, histories of fracture and disease, family history of fracture (especially hip fracture and kyphosis), and information on physical and sports activities, current smoking, alcohol consumption, and any history of dieting behav- ior. Women were defined as underweight $(\mathrm{BMI}<18.5)$, non-obese (BMI 18.5-29.9), and obese (BMI was $>30$ ). Alcohol use was defined as regular consumption at one time or more per week. Regular exercise was defined as any kind of physical activity 3 or more times per week. Bone mineral density (BMD) was measured using several methods including dual-energy X-ray absorptiometry (DXA) at the lumbar spine (L2-4) $(1.2 \%$ of total measurements), hip (0.5\%), and forearm $(27.0 \%)$, quantitative ultrasound of the calcaneus $(23.5 \%)$, and computed X-ray densitometry (CXD) at the second metacarpal (47.1\%) [16]. BMD was expressed as percentage of the average for a young adult (young adult mean, YAM) at peak bone density (20-44 years old).

\section{Statistical analysis}

Data were expressed as mean \pm SD or numbers or percentages. We used Fisher's exact test for nominal variables and unpaired $t$ test for continuous variables between two groups, and $\chi^{2}$ test among 3 or more groups for nominal variables to evaluate the differences. A multiple logistic regression analysis was used to find predictors and odds ratios (OR) with $95 \%$ confidential intervals $(95 \% \mathrm{CI})$ for bone fracture and dieting behavior before and after adjustment with characteristic variables. $P<0.05$, two-tailed, was considered statistically significant. All statistical calculations were performed with IBM SPSS Statistics ver. 18 (SPSS, Chicago, IL).

\section{Results}

In this study, we analyzed 64,809 Japanese women aged $>40$ years, consisting of $13.0 \%$ at 40 years, $8.1 \%$ at 45 years, $12.4 \%$ at 50 years, $12.6 \%$ at 55 years, $17.5 \%$ at 60 years, $20.4 \%$ at 65 years, and $15.9 \%$ at 70 years, respectively. Mean age was $56.9 \pm 9.9$ years, and mean BMI was $22.2 \pm 3.1 \mathrm{~kg} / \mathrm{m}^{2}$. Family history of kyphosis was $17.6 \%$, and family history of hip fracture was $6.3 \%$. DM was observed in $3.3 \%$, dyslipidemia in $11.9 \%$, kidney disease in $2.5 \%$, liver disease in $1.8 \%$, and thyroid disease in $4.7 \%$. Menstruation was present in $31.1 \%$. Alcohol use was positive in $33.8 \%$, and $7.9 \%$ were current smokers. Women doing regular exercise 3 or more times per week represented $19.0 \%$. A history of unexpected fall within the recent 12 months was observed in $4.0 \%$. Present and past dieting behavior (dieting group) existed in $4.1 \%$ (present dieting, $0.68 \%$; and past dieting, 3.45\%). 
The number of women with any type of past bone fracture was 13,209 (20.4\%), and bone fracture during the most recent 5 years (5-year fracture) occurred in 3421 women $(5.3 \%)$. Since we studied the relation between recent lifestyle and bone fracture, 5-year fracture was used as parameter. The prevalence of 5-year fracture was $3.4 \%$ at 40 years, $3.4 \%$ at 45 years, and $3.3 \%$ at 50 years (Fig. 1A). This parameter increased in an age-dependent manner with menopause, being $5.1 \%$ at 55 years, $5.9 \%$ at 60 years, $6.7 \%$ at 65 years, and $6.9 \%$ at 70 years (Fig. 1A).

The mean value of BMD was relatively stable before menopause, $99.9 \pm 10.1 \%$ at 40 years, $99.6 \pm 11.0 \%$ at 45 years, and $98.1 \pm 10.6 \%$ at 50 years (Fig. 1B). It decreased in an age-dependent manner thereafter, being $90.8 \pm 12.2$ at 55 years, $84.6 \pm 12.3 \%$ at 60 years, $81.2 \pm 13.3 \%$ at 65 years, and $78.7 \pm 14.4 \%$ at 70 years (Fig. 1B). In the present study, we defined osteopenia with BMD between -1SD and -2.5 SD at 40 years old $(90>$ BMD $>74 \%)$, and osteoporosis with BMD less than $-2.5 \mathrm{SD}$ at 40 years old (BMD $<74 \%)$.

The bone fracture group (5-year fracture) had significantly higher age, BMI, and prevalence of delivery, family histories of kyphosis and hip fracture, DM, dyslipidemia, kidney disease, regular exercise, fall, and osteoporosis, and had significantly lower BMD and proportion of menstruating subjects (Table 1). Present and past dieting was observed in $4.8 \%$ of the fracture group, which tended to be higher than the $4.1 \%$ rate without fracture $(p=0.055)$, although the difference was not significant (Table 1).

We examined the role of DM in bone fracture, and the subjects were categorized into groups with and without DM (Table 2). The two groups had similar family histories of kyphosis, prevalence of thyroid disease and current smoking. However, women with DM had significantly higher age, higher BMI, higher prevalence of obesity, delivery, family history of hip fracture, dyslipidemia, kidney disease, liver disease, recent history of fall, dieting and osteoporosis, and had significantly lower BMD and proportion of menstruation, alcohol use and regular exercise (Table 2). Fracture occurred in $7.6 \%$ of those with DM, significantly higher than $5.2 \%$ without DM (Table 2).

In order to analyze the role of dieting, the subjects were categorized into groups with and without dieting behavior (Table 3). The two groups had similar family histories of kyphosis and hip fracture, prevalence of dyslipidemia, kidney disease, liver disease and thy-
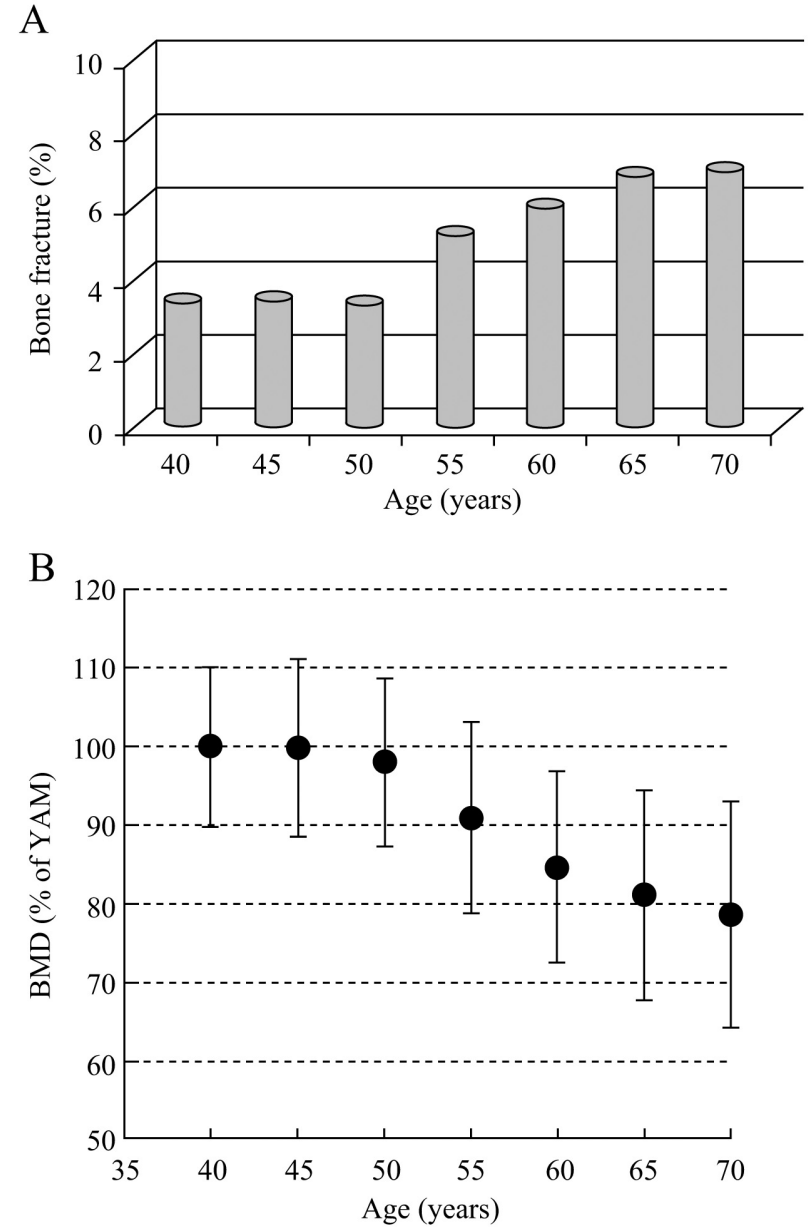

Fig. 1 A, prevalence of bone fracture (5-year fracture) and age; $\mathrm{B}$, bone mineral density and age. BMD, bone mineral density; YAM, young adult mean

roid disease. Although non-obesity (underweight and non-obese) was $95.6 \%$ in the dieting group, the women with dieting had significantly higher BMI, younger age, higher BMD, and higher rates of obesity, menstruation, $\mathrm{DM}$, alcohol use, current smoking, regular exercise, and recent history of fall, and had significantly lower rates of giving birth and osteoporosis. Bone fracture occurred in $6.1 \%$, which tended to be higher than the $5.2 \%$ rate without dieting $(p=0.055)$ (Table 3 ).

The risk factors associated with bone fracture were evaluated by logistic regression model (Table 4). Bone fracture was independently higher in every 5 -year increment of age with OR 1.05 (1.02-1.09), in family history of kyphosis with OR 1.13 (1.03-1.23), in family history of hip fracture with OR 1.27 (1.12-1.45), in DM with OR 1.26 (1.05-1.50), in kidney disease with OR 1.35 (1.11-1.65), in alcohol use with OR 1.11 (1.02- 
Table 1 Characteristics of fracture and non-fracture groups

\begin{tabular}{lccc}
\hline & Fracture group & Non-fracture group & Difference $P$ value \\
\hline Number & 3421 & 61388 & \\
Age (40/45/50/55/60/65/70) (\%) & $8.3 / 5.3 / 7.8 / 12.4 / 19.6 / 26.0 / 20.7$ & $13.3 / 8.3 / 12.6 / 12.7 / 17.4 / 20.1 / 15.6$ & $<0.001^{a}$ \\
Mean age (year) & $59.5 \pm 9.1$ & $56.8 \pm 9.8$ & $<0.001^{b}$ \\
BMI & $22.3 \pm 2.9$ & $22.1 \pm 2.9$ & $<0.01^{b}$ \\
Underweight/Non-obese/Obese (\%) & $7.1 / 91.3 / 1.6$ & $7.4 / 90.9 / 1.7$ & $0.685^{a}$ \\
Menstruation (\%) & 20.2 & 31.7 & $<0.001^{c}$ \\
Delivery (\%) & 89.7 & 88.4 & $<0.05^{c}$ \\
Family history of kyphosis (\%) & 20.8 & 17.4 & $<0.001^{c}$ \\
Family history of hip fracture (\%) & 9.0 & 6.1 & $<0.001^{c}$ \\
Diabetes mellitus (\%) & 4.7 & 3.2 & $<0.001^{c}$ \\
Dyslipidemia (\%) & 14.7 & 11.7 & $<0.001^{c}$ \\
Kidney disease (\%) & 3.4 & 2.5 & $<0.01^{c}$ \\
Liver disease (\%) & 1.8 & 1.8 & $0.992^{c}$ \\
Thyroid disease (\%) & 5.3 & 4.7 & $0.103^{c}$ \\
Alcohol use (\%) & 16.8 & 16.4 & $0.573^{c}$ \\
Current smoker (\%) & 7.8 & 7.9 & $0.919^{c}$ \\
Regular exercise (\%) & 23.6 & 18.8 & $<0.001^{c}$ \\
Recent history of fall (\%) & 7.7 & 3.8 & $<0.001^{c}$ \\
Dieting (\%) & 4.8 & 4.1 & $0.055^{c}$ \\
BMD (\% of YAM) & $84.0 \pm 14.8$ & $88.9 \pm 14.8$ & $<0.001^{b}$ \\
\hline Normal/Osteopenia/Osteoporosis (\%) & $35.0 / 40.4 / 24.6$ & $48.9 / 35.8 / 15.3$ & $<0.001^{a}$ \\
\hline Daras
\end{tabular}

Data are expressed as mean \pm SD or percentages. ${ }^{a} \chi^{2}$ test, ${ }^{b}$ Unpaired $t$ test, and ${ }^{c}$ Fisher's exact test. BMI: body mass index. Underweight: $\mathrm{BMI}<18.5 \mathrm{~kg} / \mathrm{m}^{2}$, Non-obese: $18.5 \leq \mathrm{BMI}<30 \mathrm{~kg} / \mathrm{m}^{2}$, Obese: $\mathrm{BMI} \geq 30 \mathrm{~kg} / \mathrm{m}^{2}$. BMD: bone mineral density $(\%$ of YAM), Osteopenia: $90>\mathrm{BMD} \geq 74 \%$, Osteoporosis: $\mathrm{BMD}<74 \%$.

Table 2 Characteristics of DM and non-DM groups

\begin{tabular}{|c|c|c|c|}
\hline & DM group & Non-DM group & Difference $P$ value \\
\hline Number & 2116 & 62693 & \\
\hline Age $(40 / 45 / 50 / 55 / 60 / 65 / 70)(\%)$ & $2.6 / 1.5 / 5.2 / 9.9 / 21.1 / 30.1 / 29.6$ & $13.4 / 8.3 / 12.6 / 12.7 / 17.4 / 20.1 / 15.4$ & $<0.001^{a}$ \\
\hline Mean age (year) & $62.7 \pm 7.1$ & $56.7 \pm 9.9$ & $<0.001^{b}$ \\
\hline BMI & $23.8 \pm 3.8$ & $22.1 \pm 2.9$ & $<0.001^{b}$ \\
\hline Underweight/Non-obese/Obese (\%) & $5.0 / 92.1 / 2.9$ & $8.8 / 89.2 / 2.0$ & $<0.001^{a}$ \\
\hline Menstruation (\%) & 10.0 & 31.8 & $<0.001^{c}$ \\
\hline Delivery (\%) & 90.7 & 88.4 & $<0.005^{c}$ \\
\hline Family history of kyphosis (\%) & 18.4 & 17.5 & N.S. ${ }^{c}$ \\
\hline Family history of hip fracture (\%) & 7.4 & 6.3 & $<0.05^{c}$ \\
\hline Dyslipidemia (\%) & 25.5 & 11.4 & $<0.001^{c}$ \\
\hline Kidney disease (\%) & 3.6 & 2.5 & $<0.01^{c}$ \\
\hline Liver disease $(\%)$ & 3.5 & 1.8 & $<0.001^{c}$ \\
\hline Thyroid disease (\%) & 5.5 & 4.7 & N.S. ${ }^{c}$ \\
\hline Alcohol use (\%) & 17.7 & 34.4 & $<0.001^{c}$ \\
\hline Current smoker (\%) & 8.4 & 7.9 & N.S. ${ }^{c}$ \\
\hline Regular exercise (\%) & 5.1 & 18.7 & $<0.001^{c}$ \\
\hline Recent history of fall (\%) & 6.4 & 3.9 & $<0.001^{c}$ \\
\hline Dieting (\%) & 6.0 & 4.1 & $<0.001^{c}$ \\
\hline BMD ( $\%$ of YAM) & $84.1 \pm 14.3$ & $88.8 \pm 14.8$ & $<0.001^{c}$ \\
\hline Normal/Osteopenia/Osteoporosis (\%) & $32.9 / 44.6 / 22.5$ & $48.7 / 35.7 / 15.6$ & $<0.001^{a}$ \\
\hline Fracture $(\%)$ & 7.6 & 5.2 & $<0.001^{c}$ \\
\hline
\end{tabular}

Data are expressed as mean \pm SD or percentages. ${ }^{a} \chi^{2}$ test, ${ }^{b}$ Unpaired t test, and ${ }^{c}$ Fisher's exact test. BMI: body mass index. Underweight: $\mathrm{BMI}<18.5 \mathrm{~kg} / \mathrm{m}^{2}$, Non-obese: $18.5 \leq \mathrm{BMI}<30 \mathrm{~kg} / \mathrm{m}^{2}$, Obese: $\mathrm{BMI} \geq 30 \mathrm{~kg} / \mathrm{m}^{2}$. BMD: bone mineral density $(\%$ of YAM), Osteopenia: $90>\mathrm{BMD} \geq 74 \%$, Osteoporosis: $\mathrm{BMD}<74 \%$. 
Table 3 Characteristics of dieting and non-dieting groups

\begin{tabular}{|c|c|c|c|}
\hline & Dieting group & Non-dieting group & Difference $P$ value \\
\hline Number & 2675 & 62134 & \\
\hline Age $(40 / 45 / 50 / 55 / 60 / 65 / 70)(\%)$ & 25.2/12.4/14.0/11.6/13.4/13.8/9.6 & $12.5 / 7.9 / 12.3 / 12.7 / 17.7 / 20.7 / 16.2$ & $<0.001^{a}$ \\
\hline Mean age (year) & $52.8 \pm 9.8$ & $57.1 \pm 10.3$ & $<0.001^{b}$ \\
\hline BMI & $23.3 \pm 3.8$ & $22.1 \pm 2.9$ & $<0.001^{b}$ \\
\hline Underweight/Non-obese/Obese (\%) & $4.8 / 90.8 / 4.4$ & 7.5/90.9/1.6 & $<0.001^{a}$ \\
\hline Menstruation (\%) & 47.2 & 30.4 & $<0.001^{c}$ \\
\hline Delivery (\%) & 83.1 & 88.7 & $<0.001^{c}$ \\
\hline Family history of kyphosis (\%) & 18.5 & 17.5 & $0.177^{c}$ \\
\hline Family history of hip fracture (\%) & 5.9 & 6.3 & $0.392^{c}$ \\
\hline Diabetes mellitus (\%) & 4.7 & 3.2 & $<0.001^{c}$ \\
\hline Dyslipidemia (\%) & 11.0 & 11.9 & $0.144^{c}$ \\
\hline Kidney disease $(\%)$ & 2.7 & 2.5 & $0.451^{c}$ \\
\hline Liver disease $(\%)$ & 2.1 & 1.8 & $0.236^{c}$ \\
\hline Thyroid disease (\%) & 5.3 & 4.7 & $0.145^{c}$ \\
\hline Alcohol use (\%) & 19.3 & 16.3 & $<0.001^{c}$ \\
\hline Current smoker $(\%)$ & 14.2 & 7.6 & $<0.001^{c}$ \\
\hline Regular exercise (\%) & 21.8 & 18.9 & $<0.001^{c}$ \\
\hline Recent history of fall (\%) & 6.8 & 3.9 & $<0.001^{c}$ \\
\hline BMD ( $\%$ of YAM) & $92.5 \pm 15.1$ & $88.5 \pm 14.8$ & $<0.001^{b}$ \\
\hline Normal/Osteopenia/Osteoporosis (\%) & $60.0 / 28.6 / 11.2$ & $47.7 / 38.3 / 16.0$ & $<0.001^{a}$ \\
\hline Fracture $(\%)$ & 6.1 & 5.2 & $0.055^{c}$ \\
\hline
\end{tabular}

Data are expressed as mean \pm SD or percentages. ${ }^{a} \chi^{2}$ test, ${ }^{b}$ Unpaired t test, and ${ }^{c}$ Fisher's exact test. BMI: body mass index. Underweight: $\mathrm{BMI}<18.5 \mathrm{~kg} / \mathrm{m}^{2}$, Non-obese: $18.5 \leq \mathrm{BMI}<30 \mathrm{~kg} / \mathrm{m}^{2}$, Obese: BMI $\geq 30 \mathrm{~kg} / \mathrm{m}^{2}$. BMD: bone mineral density (\% of YAM), Osteopenia: $90>\mathrm{BMD} \geq 74 \%$, Osteoporosis: $\mathrm{BMD}<74 \%$.

Table 4 Risk factors and odds ratios for bone fracture before and after adjustment

\begin{tabular}{|c|c|c|c|c|}
\hline & $\begin{array}{c}\text { Odds ratio }(95 \% \mathrm{CI}) \\
\text { before adjustment }\end{array}$ & $P$ values & $\begin{array}{l}\text { Odds ratio }(95 \% \mathrm{CI}) \\
\text { after adjustment }\end{array}$ & $P$ values \\
\hline Age (/5years) & $1.16(1.14-1.19)$ & $<0.001$ & $1.05(1.02-1.09)$ & $<0.01$ \\
\hline BMI & - & 0.685 & - & 0.527 \\
\hline Underweight & $0.95(0.83-1.09)$ & 0.463 & $1.00(0.87-1.15)$ & 0.978 \\
\hline Obesity & $0.93(0.71-1.22)$ & 0.623 & $0.85(0.63-1.13)$ & 0.258 \\
\hline Menstruation & $0.54(0.50-0.59)$ & $<0.001$ & $0.83(0.72-0.95)$ & $<0.01$ \\
\hline Delivery & $1.14(1.02-1.28)$ & $<0.05$ & $1.01(0.90-1.15)$ & 0.834 \\
\hline Family history of kyphosis & $1.25(1.14-1.36)$ & $<0.001$ & $1.13(1.03-1.23)$ & $<0.05$ \\
\hline Family history of hip fracture & $1.51(1.34-1.71)$ & $<0.001$ & $1.27(1.12-1.45)$ & $<0.001$ \\
\hline Diabetes mellitus & $1.50(1.27-1.77)$ & $<0.001$ & $1.26(1.05-1.50)$ & $<0.05$ \\
\hline Dyslipidemia & $1.30(1.18-1.43)$ & $<0.001$ & $1.04(0.93-1.16)$ & 0.476 \\
\hline Kidney disease & $1.41(1.16-1.70)$ & $<0.001$ & $1.35(1.11-1.65)$ & $<0.01$ \\
\hline Liver disease & $0.99(0.76-1.28)$ & 0.948 & $0.80(0.61-1.06)$ & 0.118 \\
\hline Thyroid disease & $1.14(0.98-1.33)$ & 0.100 & $1.03(0.88-1.22)$ & 0.714 \\
\hline Alcohol use & $1.028(0.93-1.13)$ & 0.572 & $1.11(1.02-1.20)$ & $<0.05$ \\
\hline Current smoker & $0.99(0.87-1.13)$ & 0.886 & $1.13(0.99-1.30)$ & 0.072 \\
\hline Regular exercise & $1.13(1.23-1.45)$ & $<0.001$ & $1.19(1.09-1.30)$ & $<0.001$ \\
\hline Recent history of fall & $2.14(1.87-2.45)$ & $<0.001$ & $1.98(1.72-2.23)$ & $<0.001$ \\
\hline Dieting & $1.17(0.997-1.38)$ & 0.054 & $1.25(1.05-1.49)$ & $<0.05$ \\
\hline BMD & - & $<0.001$ & - & $<0.001$ \\
\hline Osteopenia & $1.58(1.46-1.71)$ & $<0.001$ & $1.34(1.22-1.47)$ & $<0.001$ \\
\hline Osteoporosis & $2.25(2.05-2.46)$ & $<0.001$ & $1.80(1.61-2.01)$ & $<0.001$ \\
\hline
\end{tabular}

BMI: body mass index, Underweight: $\mathrm{BMI}<18.5 \mathrm{~kg} / \mathrm{m}^{2}$, Non-obese: $18.5 \leq \mathrm{BMI}<30 \mathrm{~kg} / \mathrm{m}^{2}$, Obese: $\mathrm{BMI} \geq 30 \mathrm{~kg} / \mathrm{m}^{2}$. BMD: bone mineral density ( $\%$ of YAM), Osteopenia: $90>\mathrm{BMD} \geq 74 \%$, Osteoporosis: $\mathrm{BMD}<74 \%$.

Odds ratio was calculated using multiple logistic regression analysis (confidence interval of $95 \%$ ). 
1.20), in regular exercise with OR 1.19 (1.09-1.30), in recent history of fall with OR 1.98 (1.72-2.23), in dieting with OR 1.25 (1.05-1.49), in osteopenia with OR 1.34 (1.22-1.47), and in osteoporosis with OR 1.80 (1.61-2.01). However, bone fracture was independently lower with the presence of menstruation with OR 0.83 (0.72-0.95), indicating that age, menopause, family histories of kyphosis and hip fracture, DM, kidney disease, alcohol use, regular exercise, recent history of fall, low bone mass, and dieting were risk factors for bone fracture (Table 4).

\section{Discussion}

We analyzed 64,809 Japanese women aged $>40$ years old in the Chiba bone survey between 2001 and 2009. The incidence of 5 -year fracture was stable until 50 years old, after which it increased in an age-dependent manner. This was consistent with the change in $\mathrm{BMD}$, reflecting the effect of menopause, since osteoporosis is one of the clinically important pathologies related to long-term estrogen deficiency in postmenopausal women $[3,4]$. Logistic regression analysis revealed that bone fracture was independently associated with age, family histories of kyphosis and hip fracture, DM, kidney disease, menopause, alcohol use, exercise, fall, and present and past dieting in addition to low bone mass.

Present and past dieting is unique among the risk factors for bone fracture. Since a pandemic of obesity has been observed concomitantly with the incrementally aging population in the world $[6,9]$, there is evidence of social pressure to be thin and, especially for women, of a weight bias [14]. A great number of people are exploiting weight control practices, ranging from weight loss diets to pills [17]. The relationship between dieting behavior and low bone mass has been reported [18]. Osteopenia or osteoporosis was observed in one-third of obese premenopausal women with a history of restrained eating, as was low bone mass, and they could not be explained by present dietary intake, levels of physical activity, or indices of stress, inflammation and bone turnover [18, 19]. However, the dieting group had a significantly higher BMD with several protective factors including a lower mean age and a greater proportion with menstruation in the present study.

The pathophysiological mechanism of dieting behavior in relation to bone fracture remained unclear in the present study. There are thought to be several possibilities. It is known that bone fragility is dependent not only on bone mass but also bone quality [1]. Dieting behavior might affect bone quality through insufficient intake of nutrients such as calcium and vitamin D. In addition, the dieting group had several poor lifestyle features, such as higher rates of DM, alcohol use, current smoking, regular exercise, and recent history of fall. It is possible that the combination of these poor lifestyle choices might affect bone quality.

It was also of interest that exercise (3 or more times per week) was one of the independent risks for fracture. Since a systematic review of randomized trials showed that both impact and non-impact exercise have a positive effect on BMD at the lumbar spine in preand postmenopausal women [20], and exercise such as volley ball, swimming, walking and tennis was significantly associated with high bone mass in the present study (unpublished observation), there might be differing effects of exercise on BMD and fracture. Although details of this discrepancy between BMD and fracture were obscure, it is quite likely that exercise is linked to accidental falls, a risk of fracture. Therefore, exercise should be carefully performed, and especially by the elderly.

Alcohol use has been established as a risk for fracture on the basis of a meta-analysis of postmenopausal women [21], in which no significant increase in risk was observed at a daily intake of $30 \mathrm{~g}$ or less of pure alcohol. Therefore, it was notable that alcohol use of only once or more per week presented a risk for bone fracture. Although we do not know the reason why Japanese women are susceptible to alcohol, there are at least two possible mechanisms: 1) since both height and weight of Japanese women are much lower than those seen in Western countries [22], a smaller amount of alcohol would still produce a relatively higher intake of alcohol per body weight, and 2) Asian ethnicity itself is known to be a risk for bone quality and fracture $[15,23]$. These factors might be enough to affect bone quality even with a lesser alcohol intake.

In the present study, current smoking was tended to be associated with fracture, but the risk was not significant $(p=0.072)$. Although smoking has been clearly established as a risk factor for bone future [24], the precise reason for this relatively lower risk in the present study was not clear, and further investigations in Japanese women are warranted.

In addition, DM and kidney disease were signifi- 
cantly associated with bone fracture in the present study. DM is reported to be a significant risk for bone fracture, and not only type 1 but also type 2 [25]. DM may affect osteoporotic fractures through not only diabetic complications of neuropathy and retinopathy to cause frequent falls, but also by bone metabolism itself [25]. The relationship of osteoporosis with increasing fracture risk has been well studied in not only endstage kidney disease [26], but also moderate renal dysfunction [27, 28]. Recently, it was further indicated that postmenopausal women with mild kidney disease (stage 2) are at increased risk for BMD decrease and vertebral fracture [29].

Our study has several limitations. The percentage of examinees of the total subjects was $26.1 \%$, which may not be high enough to represent all middle-aged and elderly women in Japan. Since our program was not legally binding, but rather was a recommendation for residents, some bias may have resulted from the fact that the examinees might have been well educated and interested in their own health not only in terms of osteoporosis but also lifestyle including nutrients. In addition, we used the history of fracture as an event based on structured, nurse-assisted, and self-administered questionnaires, although the fracture was not confirmed by x-ray. Since postmenopausal women have been reported to have osteoporotic fractures, especially vertebral fractures, without clinical symptoms [30], the prevalence of fracture might actually have been underestimated in the present study. Although it was also known that previous fracture presented a risk of subsequent fracture [31], bone fracture during the most recent 5 years was used as criterion, and the effect of previous fractures before this 5-year period was not evaluated. In the measurement of BMD, although DXA is a standard method, the availability of this equipment was insufficient to measure the large number of examinees, and we had to use several alternative methods as well for its measurement $[8,16,32]$.

In conclusion, bone fracture was closely associated with not only low bone mass but also the lifestyle factors of dieting behavior, exercise, and alcohol in middle-aged and elderly Japanese women. Women's health care should focus on their lifestyle to prevent bone fracture in postmenopausal osteoporosis.

\section{Contributions}

IT was responsible for the study concept and design, acquisition, analysis and interpretation of data, drafting of the manuscript, critical revision of the manuscript for important intellectual content, and statistical analysis. TTerano, MN, KK, JY, SS, and TTanaka were responsible for acquisition, analysis and interpretation of data, and critical revision of the manuscript for important intellectual content. MS was responsible for analysis and interpretation of data, and critical revision of the manuscript for important intellectual content. All authors reviewed the manuscript, and approved the final version submitted for publication.

\section{Conflict of Interest}

The authors declare that they have no conflict of interest.

\section{Acknowledgement}

We thank Sayuri Ishikawa BS, and Hiroshi Ikegami MD, (all with Chiba City Public Health Office, Chiba, Japan) for their support of this study. No individual received compensation for their contributions beyond their salaries.

\section{References}

1. NIH Consensus Panel on Osteoporosis Prevention Diagnosis, and Therapy (2001) Osteoporosis prevention, diagnosis, and therapy. JAMA 285: 785-795.

2. Seeman E, Delmas PD (2006) Bone quality--the material and structural basis of bone strength and fragility. $N$ Engl J Med 354: 2250-2261.

3. Raisz LG (2005) Pathogenesis of osteoporosis: concepts, conflicts, and prospects. J Clin Invest 115: 33183325 .
4. Seeman E (2002) Pathogenesis of bone fragility in women and men. Lancet 359: 1841-1850.

5. Harvey N, Dennison E, Cooper C (2010) Osteoporosis: impact on health and economics. Nat Rev Rheumatol 6: 99-105.

6. WHO (2011) World health statistics 2011. In. Geneva, Switzerland: World Health Organization.

7. Ministry of Health Labour and Welfare in Japan (2011) Population Trends of Japan, White Papers \& Reports 
Annual Health, Labour and Welfare Report 2009-2010. In. Tokyo, Japan.

8. Japanese Government (2000) Health and Medical Service Law for the Elderly (1982 No 80). In. Tokyo, Japan http://www.mhlw.go.jp/english/wp/policy/dl/04. pdf.

9. OECD (2011) Overweight and obese population aged 15 and above, Health - Risk factor - Overweight and obesity. In: OECD Factbook 2011: Economic, Environmental and Social Statistics Paris, France.

10. WHO (2005) Preventing chronic diseases: a vital investment. In: WHO global report. Geneva, Switzerland WHO Press.

11. WHO (2008) 2008-2013 Action plan for the global strategy for the prevention and control of non-communicable diseases. In. Geneva, Switzerland: WHO Press.

12. Han TS, Tajar A, Lean ME (2011) Obesity and weight management in the elderly. Br Med Bull 97:169-196.

13. Naughton C, Bennett K, Feely J (2006) Prevalence of chronic disease in the elderly based on a national pharmacy claims database. Age Ageing 35: 633-636.

14. Owen OR, Laurel-Seller E (2000) Weight and shape ideals: thin is dangerously in. J Appl Soc Psych 30: 979990.

15. Rachner TD, Khosla S, Hofbauer LC (2011) Osteoporosis: now and the future. Lancet 377: 12761287.

16. Yamauchi H, Fukunaga $M$, Nishikawa A, Orimo H (2010) Changes in distribution of bone densitometry equipment from 1996 to 2006 in Japan. J Bone Miner Metab 28: 60-67.

17. Weiss EC, Galuska DA, Khan LK, Serdula MK (2006) Weight-control practices among US adults, 2001-2002. Am J Prev Med 31: 18-24.

18. Shapses SA, Riedt CS (2006) Bone, body weight, and weight reduction: what are the concerns? J Nutr 136: 1453-1456.

19. Bacon L, Stern JS, Keim NL, Van Loan MD (2004) Low bone mass in premenopausal chronic dieting obese women. Eur J Clin Nutr 58: 966-971.

20. Wallace BA, Cumming RG (2000) Systematic review of randomized trials of the effect of exercise on bone mass in pre- and postmenopausal women. Calcif Tissue Int 67: 10-18.
21. Kanis JA, Johansson H, Johnell O, Oden A, De Laet C, et al. (2005) Alcohol intake as a risk factor for fracture. Osteoporos Int 16: 737-742.

22. OECD (2009) Height, Chapter 7. Health Indicators. In: OECD SOCIAL INDICATORS, Society at a Glance. Paris, France; 110-111.

23. Kanis JA (2002) Diagnosis of osteoporosis and assessment of fracture risk. Lancet 359: 1929-1936.

24. Kanis JA, Johnell O, Oden A, Johansson H, De Laet C, et al. (2005) Smoking and fracture risk: a meta-analysis. Osteoporos Int 16: 155-162.

25. Hofbauer LC, Brueck CC, Singh SK, Dobnig H (2007) Osteoporosis in patients with diabetes mellitus. $J$ Bone Miner Res 22: 1317-1328.

26. Lindberg JS, Moe SM (1999) Osteoporosis in end-state renal disease. Semin Nephrol 19: 115-122.

27. Dukas L, Schacht E, Mazor Z, Stahelin HB (2005) Treatment with alfacalcidol in elderly people significantly decreases the high risk of falls associated with a low creatinine clearance of $<65 \mathrm{ml} / \mathrm{min}$. Osteoporos Int 16: 198-203.

28. Nickolas TL, McMahon DJ, Shane E (2006) Relationship between moderate to severe kidney disease and hip fracture in the United States. J Am Soc Nephrol 17: 32233232.

29. Kaji H, Yamauchi M, Yamaguchi T, Shigematsu T, Sugimoto T (2010) Mild renal dysfunction is a risk factor for a decrease in bone mineral density and vertebral fractures in Japanese postmenopausal women. $J$ Clin Endocrinol Metab 95: 4635-4642.

30. Vogt TM, Ross PD, Palermo L, Musliner T, Genant HK, et al. (2000) Vertebral fracture prevalence among women screened for the Fracture Intervention Trial and a simple clinical tool to screen for undiagnosed vertebral fractures. Fracture Intervention Trial Research Group. Mayo Clin Proc 75: 888-896.

31. Kanis JA, Johnell O, De Laet C, Johansson H, Oden A, et al. (2004) A meta-analysis of previous fracture and subsequent fracture risk. Bone 35: 375-382.

32. Orimo H, Hayashi Y, Fukunaga M, Sone T, Fujiwara S, et al. (2001) Diagnostic criteria for primary osteoporosis: year 2000 revision. J Bone Miner Metab 19: 331337. 